\title{
OSSOS. VI. Striking Biases in the Detection of Large Semimajor Axis Trans-Neptunian Objects
}

\author{
Cory Shankman $^{1}$, J. J. Kavelaars ${ }^{1,2}$, Michele T. Bannister ${ }^{3}$, Brett J. Gladman ${ }^{4}$, \\ Samantha M. Lawler ${ }^{2}$, Ying-Tung Chen (陳英同) ${ }^{5}$, Marian Jakubik ${ }^{6}$, Nathan Kaib \\ Mike Alexandersen ${ }^{5}$, Stephen D. J. Gwyn ${ }^{2}$, Jean-Marc Petit ${ }^{8}$, and Kathryn Volk ${ }^{9}$ \\ ${ }^{1}$ Department of Physics and Astronomy, University of Victoria, Elliott Building, 3800 Finnerty Rd. \\ Victoria, British Columbia V8P 5C2, Canada; cshankm@uvic.ca \\ ${ }^{2}$ NRC-Herzberg Astronomy and Astrophysics, National Research Council of Canada, \\ 5071 West Saanich Rd., Victoria, British Columbia V9E 2E7, Canada \\ ${ }^{3}$ Astrophysics Research Centre, Queen's University Belfast, Belfast BT7 1NN, UK \\ ${ }_{5}^{4}$ Department of Physics and Astronomy, The University of British Columbia, Vancouver, BC, V6T 1Z1, Canada \\ 5 Institute of Astronomy and Astrophysics, Academia Sinica; 11F of AS/NTU Astronomy-Mathematics Building, \\ Nr. 1 Roosevelt Rd., Sec. 4, Taipei 10617, Taiwan, R.O.C. \\ ${ }^{6}$ Astronomical Institute, Slovak Academy of Science, 05960 Tatranska Lomnica, Slovakia \\ ${ }^{7}$ HL Dodge Department of Physics \& Astronomy, University of Oklahoma, Norman, OK 73019, USA \\ ${ }^{8}$ Institut UTINAM UMR6213, CNRS, Univ. Bourgogne Franche-Comté, OSU Theta F25000 Besançon, France \\ ${ }^{9}$ Lunar and Planetary Laboratory, University of Arizona, 1629 E University Blvd., Tucson, AZ 85721, USA \\ Received 2017 May 1; revised 2017 June 18; accepted 2017 June 19; published 2017 July 14
}

\begin{abstract}
The accumulating but small set of large semimajor axis trans-Neptunian objects (TNOs) shows an apparent clustering in the orientations of their orbits. This clustering must either be representative of the intrinsic distribution of these TNOs, or else have arisen as a result of observation biases and/or statistically expected variations for such a small set of detected objects. The clustered TNOs were detected across different and independent surveys, which has led to claims that the detections are therefore free of observational bias. This apparent clustering has led to the so-called "Planet 9" hypothesis that a super-Earth currently resides in the distant solar system and causes this clustering. The Outer Solar System Origins Survey (OSSOS) is a large program that ran on the Canada-France-Hawaii Telescope from 2013 to 2017, discovering more than 800 new TNOs. One of the primary design goals of OSSOS was the careful determination of observational biases that would manifest within the detected sample. We demonstrate the striking and non-intuitive biases that exist for the detection of TNOs with large semimajor axes. The eight large semimajor axis OSSOS detections are an independent data set, of comparable size to the conglomerate samples used in previous studies. We conclude that the orbital distribution of the OSSOS sample is consistent with being detected from a uniform underlying angular distribution.
\end{abstract}

Key words: Kuiper belt: general

\section{Introduction}

Examining the trans-Neptunian objects (TNOs) in the Minor Planet Center (MPC) database, Trujillo \& Sheppard (2014) noted that the then-known TNOs on orbits with semimajor axis, $a$, beyond 150 au and pericenter, $q$, beyond 30 au, have arguments of pericenter, $\omega$, clustered around $0^{\circ}$ (Trujillo \& Sheppard 2014). Many surveys are conducted near the ecliptic plane, and this results in a known bias favoring the detection of TNOs that come to pericenter near the ecliptic plane and thus have $\omega$ near $0^{\circ}$ or $180^{\circ}$. There has been no demonstrated bias that would favor detections of TNOs with $\omega$ near $0^{\circ}$ versus those at $180^{\circ}$. Batygin \& Brown (2016a) noted that the MPC TNOs with $a>250$ au also have a clustered longitude of ascending node, $\Omega$, and a longitude of pericenter, $\varpi \equiv \omega+\Omega$. Absent additional stabilizing mechanisms, gravitational perturbations from Neptune would randomize these orbital angles on relatively short timescales. If the observed clustering of orbital angles is reflective of the intrinsic TNO distribution, there must be some dynamical mechanism forcing these orbital angles to be confined to the present day. This line of reasoning has led some to hypothesize the existence of an as yet unseen giant planet in the distant solar system to explain the apparent orbital angle clustering (Trujillo \& Sheppard 2014; Batygin \& Brown 2016a). The idea that an unseen planet shapes the distant TNO region is not new and has been invoked to explain the formation of high-perihelion TNOs like (148209) $2000 \mathrm{CR}_{105}$ and (90377) Sedna (Gladman et al. 2002; Brown et al. 2004; Gomes et al. 2006; Soares \& Gomes 2013) and the possible resonant period ratios of large- $a$ TNOs (Malhotra et al. 2016).

A key premise of the most recent distant planet hypothesis, which has not yet been independently tested, is that the apparent clustering of orbital angles does not result from observing bias. It has been argued that the MPC sample is from independent surveys, thus their biases should be uncorrelated and the observed sample distribution should therefore not have strong biases for the detection of $\omega$ and $\Omega$ (Batygin \& Brown 2016a). Unfortunately, most of the TNOs in the MPC are from surveys where the discovery circumstances and survey characteristics remain unpublished, making it impossible to fully account for the observing biases in the full MPC sample.

The Outer Solar System Origins Survey (OSSOS) provides a completely independent, single-survey sample of newly discovered large- $a$ TNOs that is comparable in size to the samples used previously. OSSOS is a large program on the Canada-FranceHawaii Telescope that surveyed $170 \mathrm{deg}^{2}$ over a range of heliocentric longitudes near the ecliptic in 2013-2017. The details of the observing strategy and processing can be found in Bannister et al. (2016). The OSSOS discoveries exceed 830 TNOs with exceptionally well-determined orbits; the high-precision OSSOS astrometry allows rapid orbit determination for classification. All OSSOS discoveries brighter than the survey flux threshold were 
Table 1

The OSSOS Sample of TNOs with $a>150$ au and $q>30$ au

\begin{tabular}{|c|c|c|c|c|c|c|c|c|c|c|c|c|c|c|}
\hline $\begin{array}{l}\text { MPC } \\
\text { Desig. }\end{array}$ & $\begin{array}{l}\text { OSSOS } \\
\text { Desig. }\end{array}$ & $\begin{array}{c}a \\
(\mathrm{au})\end{array}$ & $e$ & $\begin{array}{c}q \\
(\mathrm{au})\end{array}$ & $\begin{array}{l}i \\
\left({ }^{\circ}\right)\end{array}$ & $\begin{array}{l}\Omega \\
\left(^{\circ}\right)\end{array}$ & $\begin{array}{l}\omega \\
\left(^{\circ}\right)\end{array}$ & $\begin{array}{l}\varpi \\
\left(^{\circ}\right)\end{array}$ & $\begin{array}{c}r \\
(\mathrm{au})\end{array}$ & $\begin{array}{l}m_{r} \\
\text { (discove }\end{array}$ & $\begin{array}{r}H_{r} \\
\text { ery) }\end{array}$ & $\begin{array}{l}T_{\text {peri }} \\
(J D)\end{array}$ & $\begin{array}{l}\text { No. } \\
\text { obs. }\end{array}$ & $\begin{array}{c}\text { Arc } \\
\text { (days) }\end{array}$ \\
\hline $2013 \mathrm{GP}_{136}$ & o3e39 & $150.2 \pm 0.1$ & 0.727 & 41.0 & 33.5 & -149.3 & 45.4 & -106.8 & 45.5 & 23.1 & 6.4 & 2465012 & 31 & 1566 \\
\hline $2015 \mathrm{KH}_{163}$ & o5m85 & $153.0 \pm 0.3$ & 0.739 & 39.9 & 27.1 & 67.6 & -129.2 & -61.6 & 51.7 & 24.7 & 7.6 & 2471713 & 36 & 1085 \\
\hline $2013 \mathrm{UT}_{15}$ & o3183 & $200 \pm 1$ & 0.780 & 43.9 & 10.7 & -168.0 & -107.9 & 84.1 & 61.2 & 24.1 & 6.2 & 2476001 & 38 & 1278 \\
\hline $2015 \mathrm{RY}_{245}$ & $\mathrm{o} 5 \mathrm{~s} 13$ & $226 \pm 3$ & 0.861 & 31.4 & 6.0 & -18.5 & -5.5 & -24.0 & 34.3 & 24.6 & 9.1 & 2452363 & 27 & 538 \\
\hline $2015 \mathrm{GT}_{50}$ & o5p060 & $312 \pm 2$ & 0.877 & 38.4 & 8.8 & 46.1 & 129.0 & 175.1 & 41.0 & 24.5 & 8.3 & 2451593 & 34 & 824 \\
\hline $2015 \mathrm{RX}_{245}$ & $\mathrm{o} 5 \mathrm{t} 52$ & $430 \pm 20$ & 0.894 & 45.5 & 12.1 & 8.6 & 65.2 & 73.8 & 62.4 & 24.1 & 6.1 & 2475606 & 33 & 587 \\
\hline $2015 \mathrm{KG}_{163}$ & o5m52 & $680 \pm 2$ & 0.940 & 40.5 & 14.0 & -140.9 & 32.1 & -108.8 & 41.1 & 24.3 & 8.1 & 2459752 & 29 & 739 \\
\hline $2013 \mathrm{SY}_{99}$ & uo3191 & $735 \pm 15$ & 0.932 & 50.0 & 4.2 & 29.5 & 32.2 & 61.7 & 60.9 & 24.8 & 6.8 & 2471634 & 33 & 1156 \\
\hline
\end{tabular}

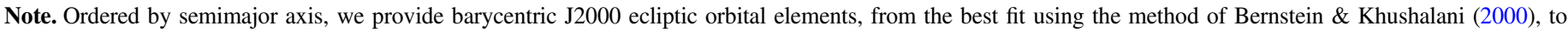

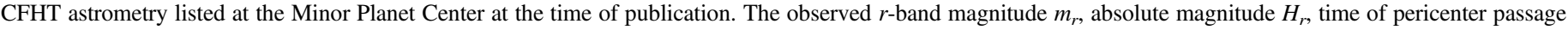

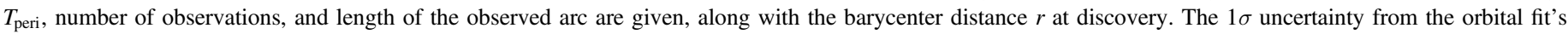
covariance matrix are listed for $a$; precisions are 0.001 for $e$ and to 0.1 for the angular elements. All digits presented are significant.

carefully and thoroughly tracked to avoid ephemeris biases (Jones et al. 2010). The sensitivity (as a function of flux and motion rate) for each OSSOS observation block is accurately determined, allowing detailed modeling of the sensitivity of OSSOS to TNO orbit distributions.

The OSSOS large- $a$ TNOs (Table 1) were all detected comparatively close to their perihelia, an expected discovery bias for large- $a$ TNOs. OSSOS detected 8 TNOs with $a>150 \mathrm{au}, q>30$ au versus the 12 TNOs from unpublished surveys contained in the MPC used in Trujillo \& Sheppard (2014), and OSSOS detected 4 TNOs with $a>250 \mathrm{au}$, $q>30$ au versus the sample of 6 MPC TNOs used in Batygin \& Brown (2016a). The OSSOS sample provides an analog to the MPC sample, while crucially also providing the detailed characterization necessary to model the observing biases affecting the detection of our discoveries.

This analysis addresses the following questions:

1. What are the observing biases, particularly those related to the orbital angles $\omega, \Omega$, and $\varpi$, in OSSOS for the $a>150$ au, $q>30$ au TNO region?

2. Is there evidence in the OSSOS sample, as has been argued for in the MPC sample of TNOs, of clustering in $\omega$ (for $a>150 \mathrm{au}), \Omega(a>250 \mathrm{au})$ or $\varpi(a>250 \mathrm{au})$ ?

3. Can we reject the null hypothesis that the intrinsic distributions of $\omega, \Omega$, and $\varpi$ are all uniform?

\section{Observations and Methods}

\subsection{OSSOS Observed Sample of Large-a TNOs}

To be consistent with Trujillo \& Sheppard (2014) and Batygin \& Brown (2016a), we use the following criteria to define our sample of TNOs: $a>150$ au and $q>30$ au. OSSOS detected eight TNOs satisfying the above criteria, four of which have $a>250 \mathrm{au}$. The discovery circumstances for two of these TNOs are described elsewhere: o3e39 (Bannister et al. 2016, $2013 \mathrm{GP}_{136}$ ) and uo3191 (Bannister et al. 2017, $2013 \mathrm{SY}_{99}$ ). The six new TNOs we present here were found during the rest of the survey: all are characterized discoveries with wellquantified detection efficiencies. The discoveries have $a$ ranging from 150 to $735 \mathrm{au}$, and all but one have $q>37 \mathrm{au}$ (Table 1).

\subsection{A Note On q Selection Criteria}

One might be tempted to impose a $q$ cut higher than 30 au on the sample to select only TNOs that do not have strong gravitational interactions with Neptune (found to be those with $q \lesssim 37$ au by Lykawka \& Mukai 2007), the argument being that TNOs with $q$ sufficiently close to Neptune will undergo evolution in $a$ and orbital orientation angles on short timescales and thus should be removed from the sample. Embedded in this argument is the assumption that the observed clustering for the sample described above does not result from observation bias. This work seeks to test that assumption, so we employ a $q$ lower limit of 30 au to be consistent with prior studies and the region where the MPC TNOs show clustering in orbital angles.

It has also been suggested that only TNOs presently dynamically stable with respect to Neptune should be used to define the sample that is examined for clustering (Batygin \& Brown 2016a), the idea being that the TNOs under consideration should be stable to perturbations from Neptune if one is to invoke an additional planet to explain their apparent clustering. If there is a massive planet in the distant solar system, the region between this planet and Neptune would be, in general, unstable. Such a planet would cause pericenter cycling and give dynamical kicks to these large- $a$ TNOs, creating a population analogous to the centaurs, which is seen in multiple simulations with a variety of additional planet candidates (Batygin \& Brown 2016b; Lawler et al. 2016; Shankman et al. 2017). TNOs beyond Neptune that are "presently" stable would not necessarily be stable in the case of an additional massive planet beyond Neptune.

In any case, if the $q$ threshold is set to a higher limit, one must still be able to explain why TNOs with $q$ further in, which should be less stable, appear clustered in the MPC sample. To reiterate, this analysis examines observational biases and looks for evidence of clustering in the OSSOS sample. Thus we select our sample using the same orbital element ranges for which arguments of clustering have been made.

\subsection{Survey Simulation of the Observability of Large-a TNOs}

OSSOS is a characterized survey with measured and reported biases. The pointing directions of the survey itself (Table 1, Bannister et al. 2016) are of key importance for the observing biases in orbital angles, as we will demonstrate. For 

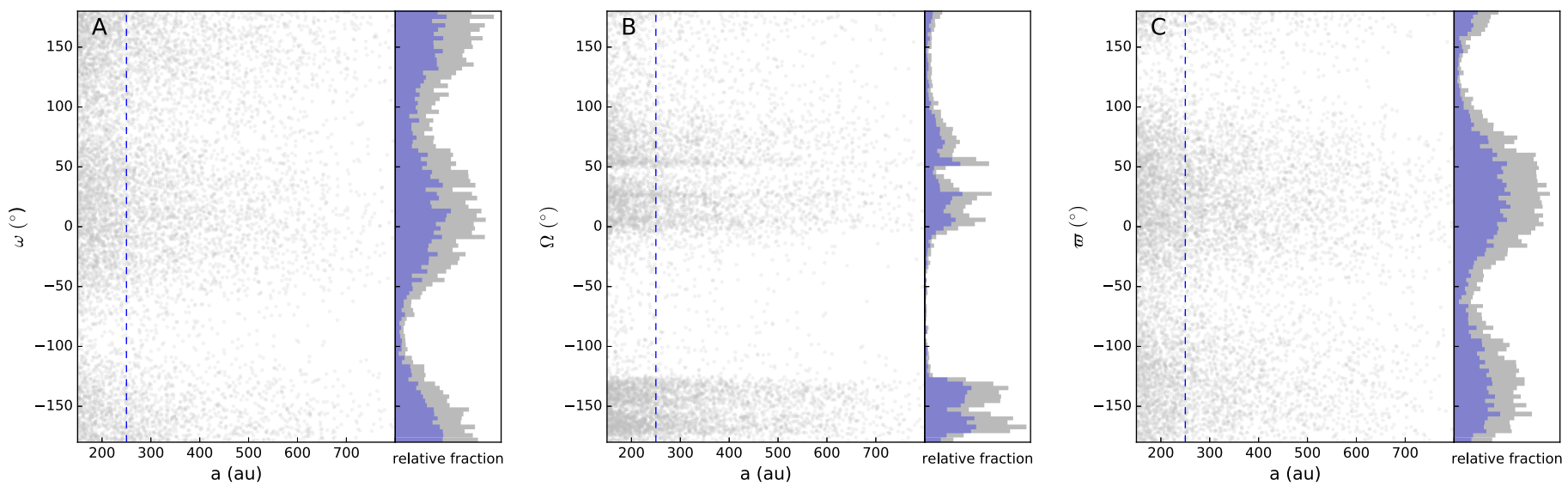

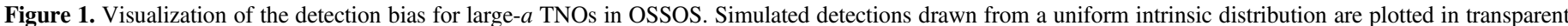

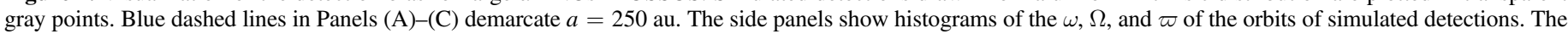

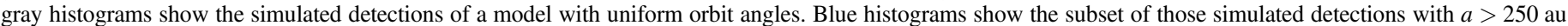
orbits. Figure 7 shows that the biases do not vary as a function of $q$.

the purposes of this analysis, we provide the relevant OSSOS TNOs (Table 1), and a full implementation of the survey simulator including an example model distribution is available by request.

We perform simulated surveys on a set of distributions of orbits with $a>150 \mathrm{au}, q>30$ au to probe the effects of the OSSOS observing biases on the detectability of TNOs in the phase space of interest. A detailed description of this established survey simulation suite can be found in Jones et al. (2006) and Petit et al. (2011), and recent examples of the use of the survey simulator in TNO studies can be found in Nesvorný (2015), Alexandersen et al. (2016), Shankman et al. (2016), and Pike et al. (2017).

We construct test distributions that fully cover ranges of orbital phase space that include the detected large- $a$ TNOs. The models tested are not intended to reproduce the observed distributions. They were designed to probe a variety of forms of distributions to test the sensitivity of the analysis to the specific choice of distribution. The models tested are combinations of distributions covering the following parameter spaces and forms.

1. $a$ : distributions spanning 150-1000 au. Distributions were either uniform in $a$ or $\propto a^{x}$, with exponents $x$ spanning 0.5-1. Distributions with an upper limit of $800 \mathrm{au}$ were also tried to test for sensitivity to the $a$ cutoff.

2. Eccentricity, $e$ : uniform from 0.7 to 0.95 . A $q$ lower limit was imposed at $30 \mathrm{au}$.

3. Inclination, $i$ : two forms were tested: (1) A uniform distribution from $0^{\circ}$ that extends up to $55^{\circ}$ (the range of the observed OSSOS sample) and (2) a distribution that scales as $\sin (i) \times$ Gaussian (as in Brown 2001). A variety of Gaussian centers (between $0^{\circ}$, and $20^{\circ}$ ) and Gaussian widths (between $5^{\circ}$ and $15^{\circ}$ ) including different combinations of centers and widths were used.

4. Absolute magnitude, $H$ : single slope from $H_{r}$ of 6 to 9.5 with a slope of 0.9. Divot and knee distributions as in Fraser et al. (2014) and Shankman et al. (2016) were also tested.

5. $\omega$, and $\Omega$ uniform from $0^{\circ}$ to $360^{\circ}$, making $\varpi$ uniform as well.
With each distribution, we conducted an OSSOS survey simulation that "detected" 10,000 simulated TNOs. These survey simulations revealed the observing biases present in the survey and show any gaps or preferences in the sensitivity to certain orbits. We found that the choice of model does not affect the conclusions about the intrinsic orbit angle distribution (see Figure 6).

\section{Results}

\subsection{Observing Bias}

Figure 1 plots the results of the survey simulation. Our simulations find that OSSOS has a range of sensitivities to and biases in different orbital parameters of TNOs. We discuss in turn our sensitivity to each angle of TNO orbit orientation. All discussions of panels in this section refer to panels in Figure 1. Panels (A)-(C) plot the orbital angles versus $a$ with histograms of the simulated detections for these angles. All statements of the sensitivity in OSSOS are made exclusively with respect to the TNO model constraints as outlined above.

$\omega$ sensitivity: OSSOS has some sensitivity to all argument of pericenter $\omega$ values, which can be seen in panels (A). Panel (A) shows that the TNOs on orbits with $\omega$ values near $0^{\circ}$ or $180^{\circ}$ are more likely to be detected. This effect arises in near-ecliptic surveys when TNOs are detected near their pericenter. The OSSOS pointings were not centered exactly around the ecliptic, with almost all the off-ecliptic coverage being north of the ecliptic. Blocks that are off the ecliptic no longer have symmetric sensitivity with $0^{\circ}$ and $180^{\circ}$ favored, but instead have only one area of reduced sensitivity, as also discussed in Sheppard \& Trujillo (2016). This lack of sensitivity is caused by the fact that some pericenter locations are not possible to detect for a survey off the ecliptic. For example, a survey that points above the ecliptic is unable to see any sky points below the ecliptic and thus has a lower sensitivity to detecting orbits that come to pericenter below the ecliptic. This effect results in less OSSOS sensitivity to TNOs with $\omega$ near $-90^{\circ}$ than near $90^{\circ}$. OSSOS still has some sensitivity to TNOs away from their pericenter due to its deep limiting magnitudes, resulting in some sensitivity to TNOs with $\omega$ near $-90^{\circ}$, as seen in panel (A). 


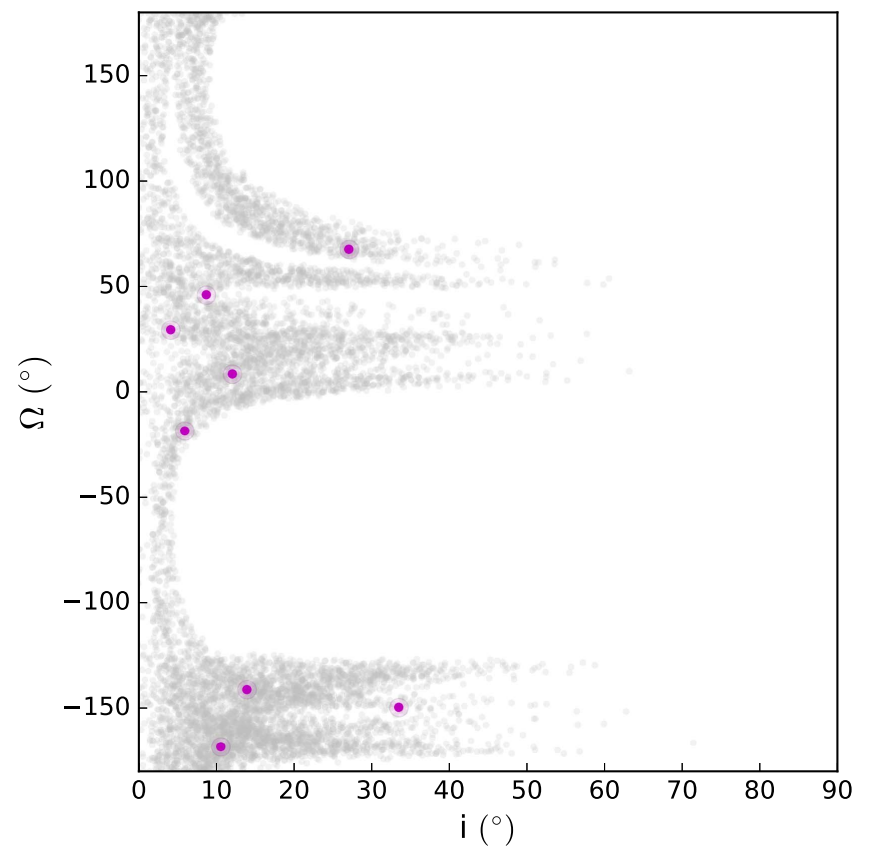

Figure 2. $\Omega / i$ sensitivity of the OSSOS project. The gray dots are the same simulated detections in Figure 1, having $a>150$ au, $q>30$ au. The OSSOS TNOs have been overplotted in magenta to show how the observed sample is affected by these strong biases.

$\Omega$ sensitivity: there exists clear and initially non-intuitive structure in the OSSOS observing bias in longitude of the ascending node $\Omega$. Panel (B) shows that there is a large and substantial gap in $\Omega$ sensitivity in the $-120^{\circ}$ to $-20^{\circ}$ range. OSSOS, due to its avoidance of the galactic plane and northern hemisphere winter, has virtually no capability for detecting large- $a$ TNOs with $\Omega$ between $-120^{\circ}$ and $-20^{\circ}$. Figure 2 shows that this structure arises from a coupling of sensitivity in $\Omega$ and $i$. This striking effect is a simple result of geometry. The $\Omega$ and $i$ angles define the plane of the TNO's orbit. In order for the TNO to be detectable by a survey, its plane must intersect the area of sky being observed. For inclined orbits to have a high chance of being detectable in an ecliptic survey, the ascending or descending node must be in the same direction as the survey's pointing. Each of the horizontal spikes in Figure 2 indicates the location of an ascending or descending node that is aligned with one of the OSSOS pointings. The bias structure curves horizontally as inclinations go toward $0^{\circ}$, when orbits become ecliptic grazing, and can thus be seen at more points across their orbit in ecliptic surveys. We plot the OSSOS detections in Table 1 in panel Figure 2 to show that they follow this bias-induced distribution of $\Omega$ and $i$.

$\varpi$ sensitivity: the sensitivity to detecting longitude of pericenter $\varpi$ is a combination of the sensitivities to detecting $\Omega$ and $\omega$. The sensitivity is a double peaked distribution, with less sensitivity to $\varpi$ in the range of $110^{\circ}-160^{\circ}$ (see Panel (C)). OSSOS has some sensitivity to all $\varpi$ values and there is no striking structure in the $\varpi$ sensitivity other than the two broad peaks roughly separated by $180^{\circ}$ of longitude.

We examined the three above biases for both the $a>150$ au and $a>250$ au regions to explore if the sensitivity changes with $a$. We found that the observing biases are the same for the two regions, which can be seen by comparison of the blue and gray histograms in Figure 1. Once a TNO has a sufficiently large orbit, it is only detectable near pericenter. This bias strongly affects the expected detection of orbital angles as we have shown. The bias structure does not change as a function of increasing $a$ because the bias is a result of the fact that the TNOs are only detectable near pericenter.

Although the biases we demonstrate are specific to OSSOS, all TNO surveys will have complicated detection biases like those shown in this work. Without publishing characterizations of the survey pointings, these complex and often non-intuitive biases cannot be accounted for, and may lead to incorrect assumptions about the intrinsic population.

\subsection{Angle Clustering in the OSSOS Sample}

Having examined the biases of OSSOS, we now examine the detected OSSOS sample (Table 1) for evidence of a clustering in the orbital angles for the large- $a$ TNOs, as first noted in the MPC data set by Trujillo \& Sheppard (2014) and Brown \& Batygin (2016). We consider the OSSOS sample independently, examining the distributions of the OSSOS TNOs with no a priori expectations about clustering.

A visual examination of the orbital distribution (see Figure 3) shows relatively little evidence of clustering of $\omega$, even in the observationally biased $a>150$ au OSSOS sample. The eight OSSOS TNOs are found to be distributed across the full range of $\omega$ values (Figure 3 panel (A)). We demonstrated in Section 3.1 that OSSOS has some sensitivity to all $\omega$ values; this sensitivity is reflected in the broad distribution of detected $\omega$ values. For each orbital angle, we test the hypothesis that the OSSOS sample can be detected from a uniform intrinsic distribution. To do this, we compare the observed distributions to the survey simulator biased models described above (Figure 1). We test the null hypothesis using Kuiper's test (e.g., see Fisher 1995; Pewsey et al. 2013), which is closely related to the Kolmogorov-Smirnov test, but is invariant to cyclic transformations of the test variable. The test is thus wellsuited to problems with cyclic variables, as is the case for angles like $\omega, \Omega$, and $\varpi$. The eight TNOs are statistically consistent (i.e., $53 \%$ of bootstrapped model subsamples have a larger Kuiper's test distance than the observed sample to the parent model: the hypothesis is rejectable at $47 \%$, i.e., not rejectable) with being detected from an intrinsic uniform distribution of $\omega$ values. Our results hold for all tested intrinsic models described in Section 2.3.

We now examine the $\Omega$ and $\varpi$ distributions for the OSSOS TNOs with $a>250 \mathrm{au}$. We find that the $\Omega$ values for three of the TNOs are distributed near $25^{\circ}$ with the fourth isolated (Figure 3 panel (B)). OSSOS had effectively no sensitivity to TNOs with $\Omega$ between $-120^{\circ}$ and $-20^{\circ}$, and had poor sensitivity to TNOs with $\Omega$ between $115^{\circ}$ and $165^{\circ}$ (see Figure 3 panel (B) histogram). Unsurprisingly, OSSOS did not detect TNOs in regions of limited or no sensitivity. Using Kuiper's test, we find that the OSSOS detections are statistically consistent (rejectable at $61 \%$, i.e., not rejectable) with being detected from an intrinsic uniform distribution of $\Omega$ values. We find that the $\varpi$ values cover a large range, with only two values near each other. As with $\omega$ and $\Omega$, the OSSOS TNO $\varpi$ values are consistent (rejectable at $62 \%$, i.e., not rejectable) with being detected from an intrinsic uniform distribution of $\varpi$ values. 

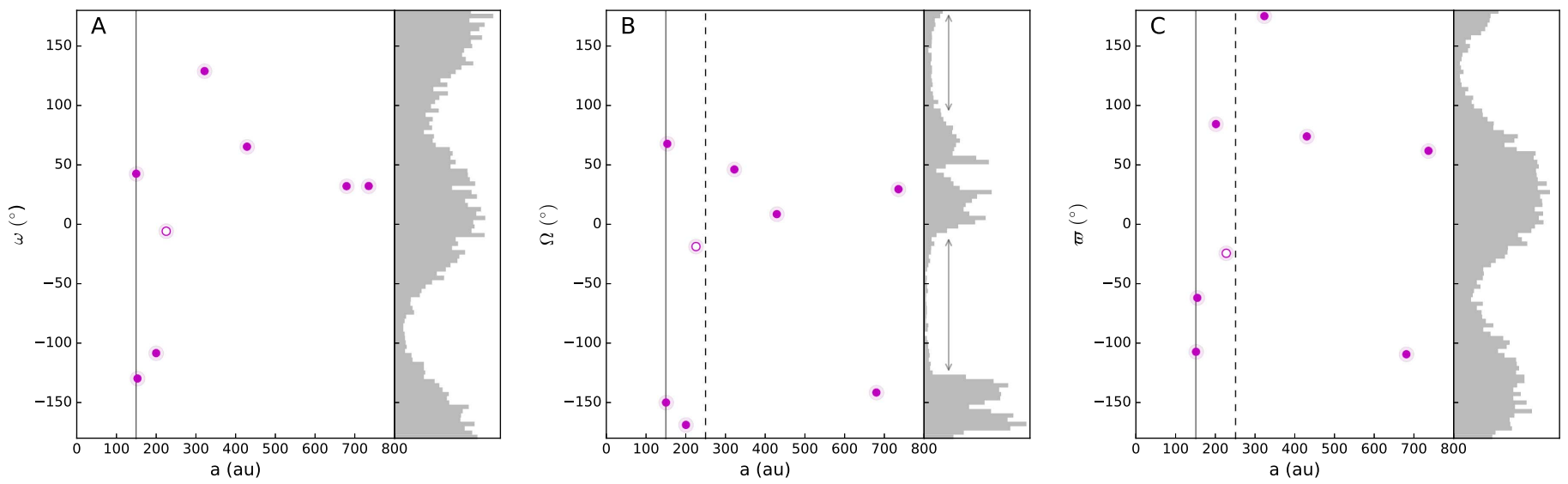

Figure 3. The OSSOS detections satisfying $a>150$ au and $q>30$ au are shown with magenta points. Open circles indicate TNOs with $q<37$ au $-\mathrm{a}$ threshold cited to demarcate the region of stability from Neptune perturbations (Lykawka \& Mukai 2007). Solid vertical lines mark 150 au and dashed lines mark 250 au. Histograms repeat the OSSOS sensitivity in each parameter as in Figure 1. The double-sided arrows in the Panel (B) histogram mark the $\Omega$ ranges where OSSOS has low sensitivity due to the survey's bias, and thus detections are unlikely.
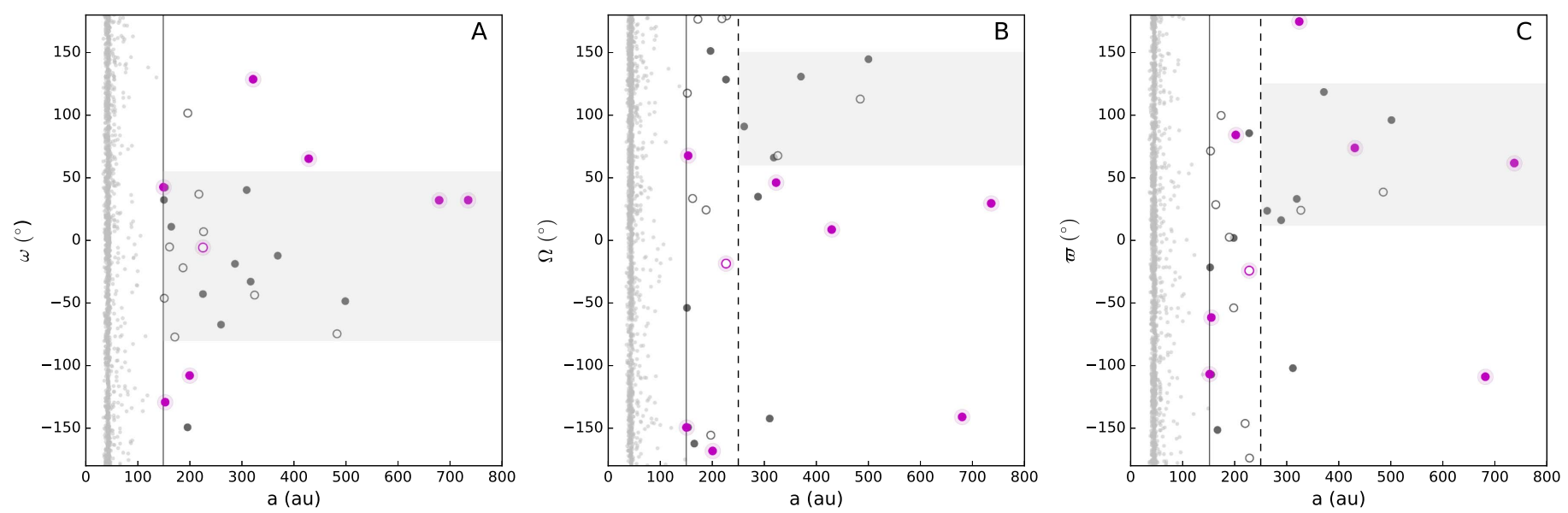

Figure 4. Plots of the orbits of the OSSOS and MPC samples for $\omega, \Omega$ and $\varpi$ vs. $a$. The MPC sample $\left(2014 \mathrm{FE}_{72}\right.$ with $a=2155$ au, $q=36$ au, $i=20^{\circ}, \omega=134^{\circ}$, and $\Omega=-23^{\circ}$ has been excluded from these plots because it interacts with galactic tides (Sheppard \& Trujillo 2016)) has been selected with $q>30$ au. OSSOS detections are shown in magenta points. MPC TNOs are plotted in transparent gray points, which are larger for $a>150$ TNOs. There are no OSSOS discoveries in the $a>150$ au MPC gray points. The solid vertical lines mark 150 au and the dashed lines mark 250 au. The gray shaded regions indicate the regions of apparent clustering in the MPC sample proposed by previous authors. As in Figure 3, open circles indicate TNOs with $q<37$ au, showing how a stability argument might affect the argument for clustering. It is clear from this view that the MPC TNOs with $q$ between 30 and 37 au still appear to cluster in $\omega$ (for $a>150$ au) and $\Omega$ and $\varpi$ (for $a>250 \mathrm{au}$ ), despite the fact that interactions with Neptune would prevent shepherding by an external planet.

We conclude that the independent OSSOS sample shows no evidence for intrinsic clustering in the $\omega, \Omega$, or $\varpi$ distributions of TNOs.

\subsection{OSSOS and MPC Sample Comparison}

We now compare the OSSOS sample (known biases) to the MPC sample (unknown biases) to examine the broader question of clustering in the known TNOs. Figure 4 plots the eight OSSOS TNOs and the MPC TNOs satisfying $a>150$ au and $q>30$ au as reported by the MPC in 2017 April.

Figure 4 panel (A) shows clearly that the apparent clustering in $\omega$ that has been noted in the MPC sample is not present in the OSSOS sample, despite the OSSOS survey biases against $\omega= \pm 90^{\circ}$. Where the MPC sample is contained within roughly $50^{\circ}$ of $0^{\circ}$, the OSSOS sample spans all values and has as many TNOs inside the apparent clustering region (gray shading of
Figure 4 panel (A)) as outside. The OSSOS TNOs that are outside the apparent clustering region have a variety of semimajor axes and all have $q>37$ au. With the addition of the OSSOS sample and a few recently discovered TNOs in the MPC sample, the argument for a clustering of $\omega$ in the detected TNOs has been substantially weakened.

There is no overlap between the OSSOS sample and the $\Omega$ clustering region of TNOs with $a>250$ au noted in Batygin \& Brown (2016a), with all four OSSOS detections outside the clustered band. The four $a>250$ au OSSOS detections span a range of $a$ values and all have $q>37 \mathrm{au}$. If one were to consider the three OSSOS detections with $\Omega$ between $0^{\circ}$ and $50^{\circ}$ to be part of the clustered grouping, the clustering would then span approximately $150^{\circ}$. Sheppard \& Trujillo (2016) noted that their recent discoveries began to erode the signal of clustered $\Omega$ in the large- $a$ TNOs; the four OSSOS detections outside the previously reported band continue this trend of 


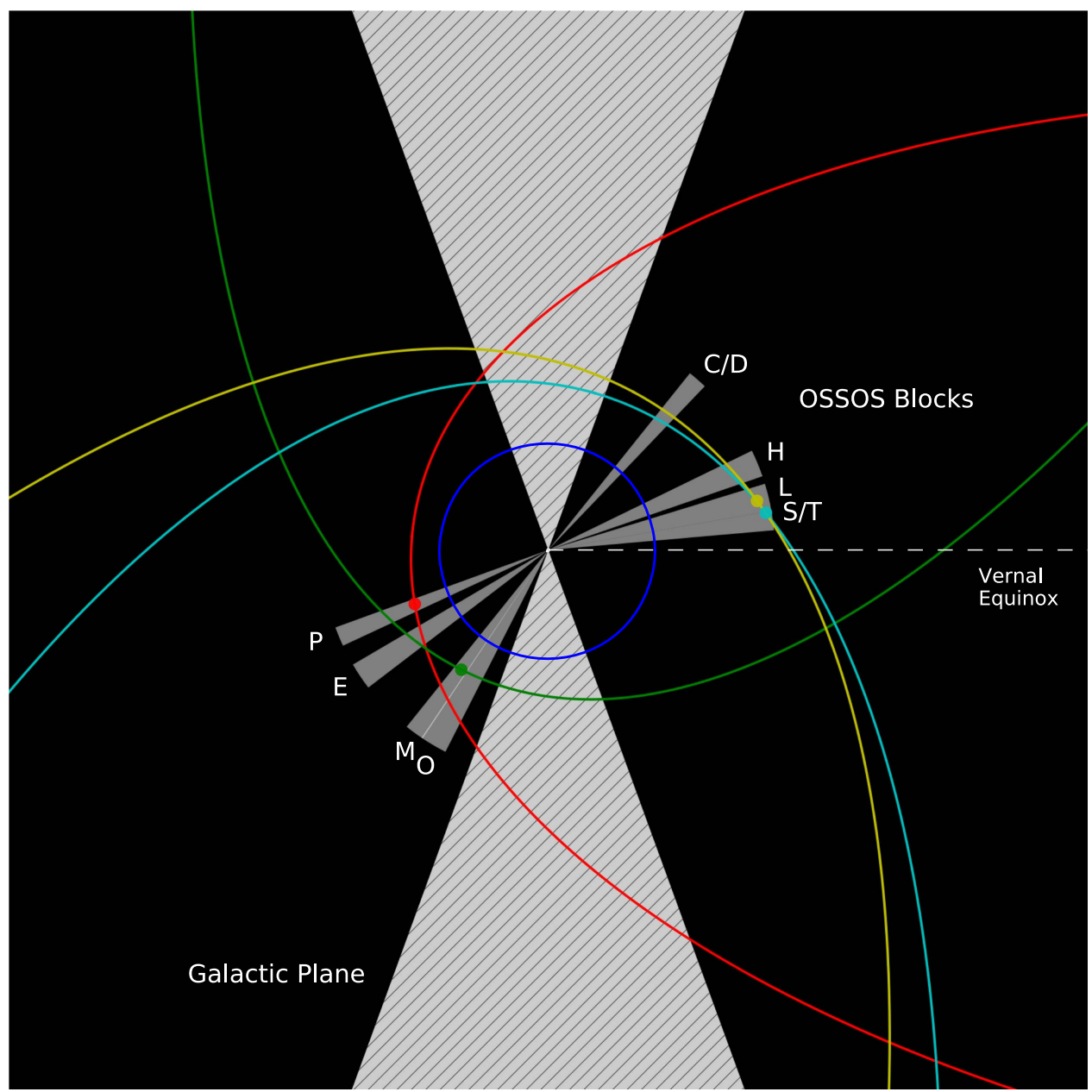

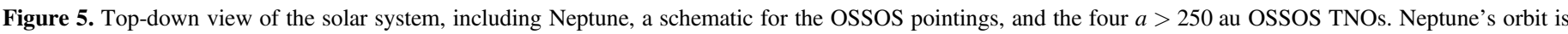

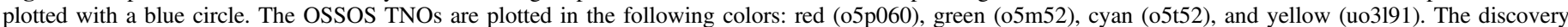

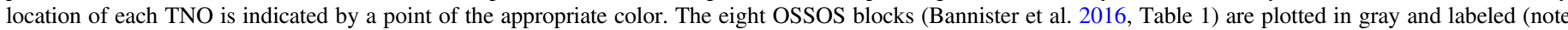

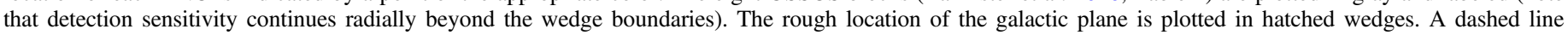
indicates the direction of the vernal equinox,therefore the upper right quadrant is the September-to-November opposition direction.

eroding the signal. This would be expected if the original signal results from a combination of small number statistics and observing bias.

Two of the OSSOS $a>250$ au TNOs with $\varpi$ near $70^{\circ}$ are within the Batygin \& Brown (2016a) proposed "anti-aligned" cluster. The third, $05 \mathrm{~m} 52$, with a $\varpi$ of $-110^{\circ}$, is approximately $180^{\circ}$ away, and would fall in the subsequently postulated "aligned" cluster (Brown \& Batygin 2016; Sheppard \& Trujillo 2016). Discoveries at these values of $\varpi$ are unsurprising in OSSOS, as the observing bias favors detections with $\varpi$ at these longitudes (Figure 1 panel (C)). The final OSSOS detection, o5p060, however, is approximately $90^{\circ}$ away from each of these proposed clustering regions.

\section{Discussion and Summary}

We find no evidence in the OSSOS sample for the $\omega$ clustering that was the impetus for the current additional planet hypothesis (Trujillo \& Sheppard 2014). The OSSOS $\omega$ distribution cannot reject the null hypothesis that the underlying distribution is random, once the biases are taken into account. Our analysis of the OSSOS survey bias and our detections do not directly address the question of why the majority of presently known MPC TNOs are clustered around $\omega$ of $0^{\circ}$. We suggest that this clustering is the result of a combination of observing bias and small number statistics, though we cannot test this without published characterizations of the surveys that detected these TNOs. It must be the case that OSSOS and the other surveys that compose the MPC sample have observed the same intrinsic distribution. OSSOS found TNOs across all values despite being most sensitive to TNOs in the clustering band (near $0^{\circ}$ ). The OSSOS detections go beyond the relatively tight clustering seen in the observed sample, and the OSSOS distribution is consistent with a uniform intrinsic $\omega$ distribution. This result calls into question the idea of a clustering of $\omega$ around $0^{\circ}$ in the intrinsic distribution of $a>150$ au $q>30$ au TNOs.

We have demonstrated that $\Omega$ biases are strong and very present in surveys such as OSSOS. These complex biases must also exist in the surveys that compose the MPC sample; it is not sufficient to state that the surveys are independent and therefore the biases must have averaged out. There have been only a handful of surveys that have detected such large- $a$ TNOs, and the biases from these surveys have shaped the MPC sample in unknown ways. There is a large gap in the known TNO $\Omega$ distribution for both OSSOS and MPC 
samples. This gap occurs precisely where OSSOS has no sensitivity due to the survey's construction. In OSSOS, this gap is driven by weather patterns at the Canada-FranceHawaii Telescope and pointing choices that avoid the dense star fields of the galactic plane. Figure 5 provides a visual representation that demonstrates the nature of these biases. OSSOS observed in the northern spring (April-May) and fall (September-November), and has virtually no sensitivity to orbits that intersect the ecliptic at other times of year. TNO surveys have been conducted from a limited number of locations and are subject to similar constraints as OSSOS. In particular, the best conditions (and thus deepest coverage) are in these months. It is therefore possible that the surveys that detected the MPC sample contain these same biases and therefore the gap in the detected $\Omega$ distribution may result simply from pointing constraints.

It has been argued that no $\omega$ or $\varpi$ biases are seen in the close-in TNOs in the MPC sample and thus there should be no biases in the large- $a$ sample. Unique biases arise from the fact that the large- $a$ TNOs are only detected near their pericenters. The lack of observed clustering in the close-in TNOs cannot simply be extended to conclude that the large- $a$ TNOs lack bias. To verify this via simulation, we examined the OSSOS sensitivity to close-in TNOs, as in Section 2.3. OSSOS has equal sensitivity for all values of $\omega$ and $\varpi$, in contrast to the striking biases observed for the large- $a$ TNOs (see Figure 8).

Much attention has been given to the appearance of clustering of "aligned" and "anti-aligned" orbits in physical space (apparent clustering of $\varpi$ ), which is founded on the assertion that there is no bias in the detection of $\varpi$. However, we have shown that for TNOs detected near pericenter, the detection of $\varpi$ is also driven strongly by where one looks in the sky. Historical surveys will also show bias favoring detections in the aligned and antialigned directions due to the prevalence of large-scale surveys occurring (and having the best weather) in the spring and fall. In order to efficiently detect large- $a$ TNOs outside the aligned and anti-aligned clusters, one would need large survey coverage from June to August and December to February. We posit that two clusters $180^{\circ}$ apart are the natural outcome of seasonal weather biases when observing a highly eccentric population for which detection is only possible close to pericenter. The observed TNOs therefore do not require the existence of a non-uniform intrinsic distribution (the impetus of the additional planet hypothesis). Additionally, the MPC sample's $\omega$ values are all near $0^{\circ}$ and as we have shown, the detected $\Omega$ distribution is strongly set by the geometry of the pointing directions. The clustering seen in $\varpi$ in the MPC sample therefore likely results from adding numbers near $0^{\circ}$ to strong observing biases present in the $\Omega$ distribution. The apparent $\varpi$ clustering seen in the MPC sample thus cannot be taken to be independent of bias.

While OSSOS was primarily sensitive to orbits with $\varpi$ near the region of the MPC sample clustering (see Figure 1), it still found one quarter of its sample away from this region where the sensitivity is low. Despite the reduced sensitivity to such orbits, OSSOS detected o5p060 with a $\varpi$ that produces an orbit orthogonal to the suggested clustering axis. This suggest that there must be a large population of TNOs on similar orbits (of order ten thousand $\left.{ }^{10}\right)$, or that the detection of 05 p060 was

\footnotetext{
${ }^{10}$ Survey simulations show that approximately 13,000 TNOs with $H_{r}<9$ on orbits within the uncertainty of 05p060's orbit are required to explain the detection of $05 \mathrm{p} 60$ in OSSOS.
}

anomalous. In either case, the existence of o5p060 with $a$ of $314 \mathrm{au}, q$ of $38 \mathrm{au}$, and $\varpi 90^{\circ}$ away from the clustering region provides evidence of a population that would refute a simplistic interpretation of the extra-planet hypothesis (Brown \& Batygin 2016) in which only anti-aligned orbits can survive.

One might be tempted to choose a different pericenter sample cut, pushing the limit away from 30 au. Setting a limit of 40 au would remove two of the six TNOs noted by Batygin \& Brown (2016a) to cluster and would remove one of the four TNOs in the OSSOS sample. If one is to argue that a dynamical effect causes the clustering of only the TNOs with $q$ greater than a limit of $40 \mathrm{au}$ (or any other choice), it must then be explained why the MPC sample of TNOs with $40<q<30$ au also appears to cluster, if the effect is not caused by observing bias.

We have shown that there are strong and striking biases in the detection of the orbital angles present in OSSOS. There is no evidence for clustering in the OSSOS sample when considered alone, and when OSSOS is folded into the MPC sample the arguments for clustering in the detected TNOs erode. The first large independent sample shows no evidence for the hypothesized intrinsic clustering. While the idea of there being a larger-than-dwarf-scale planet in the outer solar system as a mechanism to create the $q$-detached TNOs is still plausible, the evidence that there is currently a super-Earth or larger planet confining the large- $a$ TNOs is in doubt.

This project was funded by the National Science and Engineering Research Council and the National Research Council of Canada. This research used the facilities of the Canadian Astronomy Data Centre operated by the National Research Council of Canada with the support of the Canadian Space Agency. C.J.S. gratefully acknowledges support from the NSERC CGS Fellowship. M.T.B. appreciatively acknowledges support from UK STFC grant ST/L000709/1. S.M.L. gratefully acknowledges support from the NRC Canada Plaskett Fellowship. M.J. acknowledges the support from the Slovak Grant Agency for Science (grant No. 2/0031/14). The authors recognize and acknowledge the sacred nature of Maunakea, and appreciate the opportunity to use data observed from the mountain. We thank the dedicated staff of the Canada-France-Hawaii Telescope (CFHT). CFHT is operated by the National Research Council of Canada, the Institute National des Sciences de l'Univers of the Centre National de la Recherche Scientifique of France, and the University of Hawaii, with OSSOS receiving additional access due to contributions from the Institute of Astronomy and Astrophysics, Academia Sinica, Taiwan. Observations were obtained with MegaPrime/MegaCam, a joint project of CFHT and CEA/DAPNIA. We would like to thank the anonymous referee for the discussion and suggestions, which have helped us to improve this work.

Facilities: NRC, CFHT (MegaPrime).

Software: Mercury, Python.

\section{Appendix Additional Figures}

The Appendix contains Figures 6-8. 

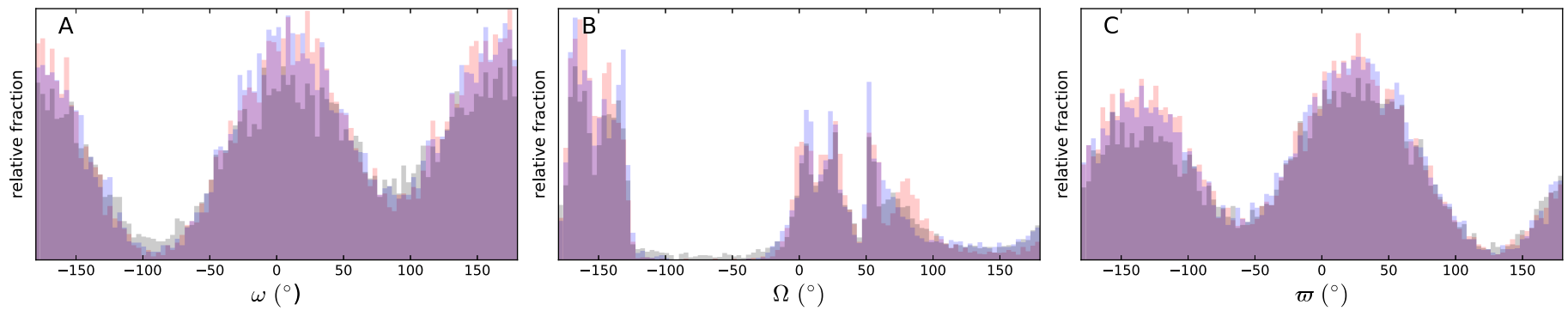

Figure 6. Histograms of the sensitivity of OSSOS, as in Figure 1, to three different models of orbit distributions (plotted with transparencies). These three models explore different $a$ and $i$ distributions. One model has flat $a$ and $i$ distributions with $a$ spanning 150-800 au and $i$ up to $55^{\circ}$. The other two models have power-law $a$ distributions and $i$ distributions that are drawn from $\sin (i) \times$ Gaussian distributions with different centers and widths. This shows that the results hold in general across different choices of model distributions.
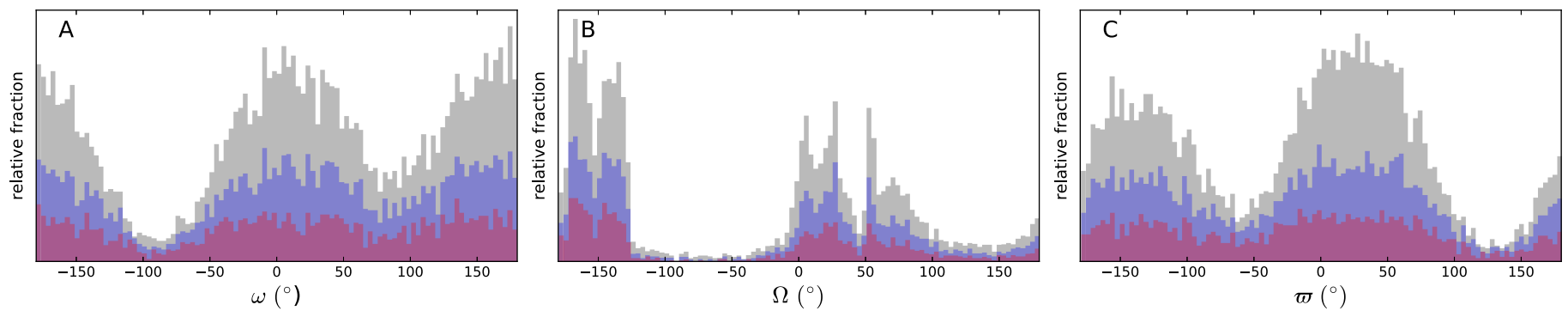

Figure 7. Histograms of the sensitivity of OSSOS as in Figure 1. The gray histogram shows all simulated detections, the blue histogram shows those with $q<37$ au, and the magenta histogram shows TNOs with an even lower cutoff of $q<34$ au. The form of the bias is the same for all $q$ cuts, but the bias is more pronounced for the largest $q$ TNOs.
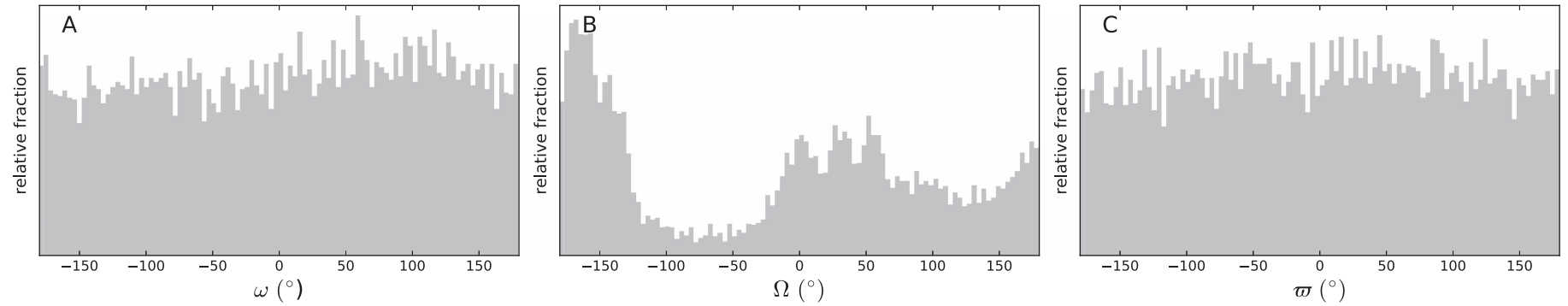

Figure 8. Plots of the OSSOS sensitivity to a model orbit distribution with close-in TNOs. The model has both $a$ and $q$ between 30 and 50 au. The strong biases observed in $\omega$ and $\varpi$ for the large- $a$ TNOs (Figure 2) are not present for this close-in population, which has near equal sensitivity to detecting TNOs with all $\omega$ and $\varpi$ values. The biases seen in Figure 1 arise from detecting TNOs near pericenter, and thus are not present for close-in TNOs that can be detected at any point in their orbit. The biases in $\Omega$ arise from the geometry of orbits intersecting pointing locations, and thus are still present in the close-in sample.

\section{References}

Alexandersen, M., Gladman, B., Kavelaars, J. J., et al. 2016, AJ, 152, 111

Bannister, M. T., Kavelaars, J. J., Petit, J.-M., et al. 2016, AJ, 152, 70

Bannister, M. T., Shankman, C., Volk, K., et al. 2017, AJ, 153, 262

Batygin, K., \& Brown, M. E. 2016a, AJ, 151, 22

Batygin, K., \& Brown, M. E. 2016b, arXiv:1610.04992

Bernstein, G., \& Khushalani, B. 2000, AJ, 120, 3323

Brown, M. E. 2001, AJ, 121, 2804

Brown, M. E., \& Batygin, K. 2016, ApJL, 824, L23

Brown, M. E., Trujillo, C., \& Rabinowitz, D. 2004, ApJ, 617, 645

Fisher, N. I. 1995, Statistical Analysis of Circular Data (Cambridge: Cambridge Univ. Press), 71

Fraser, W. C., Brown, M. E., Morbidelli, A., Parker, A., \& Batygin, K. 2014, ApJ, 782, 100

Gladman, B., Holman, M., Grav, T., et al. 2002, Icar, 157, 269
Gomes, R. S., Matese, J. J., \& Lissauer, J. J. 2006, Icar, 184, 589 Jones, R. L., Gladman, B., Petit, J.-M., et al. 2006, Icar, 185, 508

Jones, R. L., Parker, J. W., Bieryla, A., et al. 2010, AJ, 139, 2249

Lawler, S. M., Shankman, C., Kaib, N., et al. 2016, arXiv:1605.06575

Lykawka, P. S., \& Mukai, T. 2007, Icar, 192, 238

Malhotra, R., Volk, K., \& Wang, X. 2016, ApJL, 824, L22

Nesvorný, D. 2015, AJ, 150, 73

Petit, J.-M., Kavelaars, J. J., Gladman, B. J., et al. 2011, AJ, 142, 131

Pewsey, A., Neuhäuser, M., \& Ruxton, D. 2013, Circular Statistics in R Series A Statistics in Society, 179, 1132

Pike, R. E., Lawler, S., Brasser, R., et al. 2017, AJ, 153, 127

Shankman, C., Kavelaars, J., Gladman, B. J., et al. 2016, AJ, 151, 31

Shankman, C., Kavelaars, J. J., Lawler, S. M., Gladman, B. J., \& Bannister, M. T. 2017, AJ, 153, 63

Sheppard, S. S., \& Trujillo, C. 2016, AJ, 152, 221

Soares, J. S., \& Gomes, R. S. 2013, A\&A, 553, A110

Trujillo, C. A., \& Sheppard, S. S. 2014, Natur, 507, 471 